\title{
De la drachme au denier
}

\section{Catherine Grandjean}

\section{OpenEdition \\ Journals}

Édition électronique

URL : http://journals.openedition.org/ress/204

DOI : $10.4000 /$ ress.204

ISSN : 1663-4446

\section{Éditeur}

Librairie Droz

\section{Édition imprimée}

Date de publication : 1 juillet 2007

Pagination : 19-30

ISBN : 978-2-600-01155-6

ISSN : 0048-8046

Référence électronique

Catherine Grandjean, « De la drachme au denier », Revue européenne des sciences sociales [En ligne], XLV-137 | 2007, mis en ligne le 01 juillet 2010, consulté le 30 avril 2019. URL : http:// journals.openedition.org/ress/204; DOI : 10.4000/ress.204 


\section{Catherine GRANDJEAN}

\section{DE LA DRACHME AU DENIER}

\section{INTRODUCTION}

L'époque hellénistique n'intéresse guère les spécialistes d'histoire économique du monde grec depuis que la recherche en ce domaine est marquée par les idées de Moses I. Finley. Ce dernier éprouvait peu d'intérêt et d'estime pour cette période dont il a écrit qu'elle était lassante, monotone, et souvent ignoble, tissée de guerres incessantes, de mauvaise foi et d'assassinats ${ }^{1}$. Plus sérieusement, Finley estimait que, de l'époque archaïque grecque à l'Antiquité tardive, l'économie antique avait visé avant tout l'autosuffisance, que la ressource essentielle y était l'agriculture et que le commerce, la banque et la monnaie y avaient joué un rôle négligeable. Puisqu'il envisageait l'histoire de l'Antiquité comme un tout où les permanences l'emportaient sur les évolutions, il eut tendance à privilégier l'étude des commencements, c'est-à-dire celle des cités archaïques et classiques.

Malgré la distance prise aujourd'hui par bien des spécialistes avec les positions de Finley notamment en ce qui concerne la prétendue unité de l'économie antique et son orientation quasi exclusive vers l'autosuffisance, les grands débats actuels en histoire économique du monde grec restent pour l'essentiel centrés sur les cités archaïques et classiques.

Pour la période hellénistique, la référence demeure donc, en l'absence de travail plus récent, la synthèse ancienne de Rostovtseff parue en 1941, ouvrage d'une érudition et d'une puissance intellectuelle remarquables ${ }^{2}$. La lecture de la période par l'historien russe repose sur l'idée d'une opposition entre une haute époque hellénistique très prospère et une basse époque marquée par la pénurie monétaire. Alexandre aurait favorisé la croissance en injectant dans l'économie une monnaie abondante et solide frappée avec les trésors des souverains perses. La monnaie se serait alors imposée dans tout l'Orient, remplaçant largement le troc, jusqu'en Inde et en Afghanistan, les banques se développant avec la monnaie et le commerce, tandis qu'on multipliait les routes et les infrastructures portuaires, dans un climat international relativement serein. Ensuite, aux $\mathrm{II}^{\mathrm{e}}$ et $\mathrm{I}^{\mathrm{er}}$ siècles avant notre ère, les guerres incessantes entre les souverains des royaumes nés du morcellement de l'Empire d'Alexandre, les habitudes de thésaurisation des monarques hellénistiques, des temples et particuliers, les ponctions de métaux précieux par les Romains (butin et fiscalité), auraient provoqué une pénurie monétaire. Faute de

\footnotetext{
1 Finley (1971), p. 154.

2 Rostovtseff (1989).
} 
métaux précieux, les Grecs auraient alors multiplié les émissions monétaires en bronze, puis la monnaie romaine aurait remplacé les monnaies hellénistiques et tout serait alors rentré dans l'ordre, le denier remplaçant la drachme.

Rostovtseff reconnaissait volontiers que faute de données globales sur la valeur et le volume des monnaies frappées, sur les prix et sur les taux d'intérêt, son tableau de la basse époque hellénistique était conjectural. Il ne voyait toutefois pas d'autre façon d'expliquer le développement général de la monnaie de bronze pendant la période.

Les progrès de la recherche invitent évidemment à retoucher ce tableau dont le modernisme n'est plus guère de mise aujourd'hui, même si la vision statique et primitiviste de Finley et de ses épigones est de plus en plus contestée elle aussi ${ }^{3}$. La prétendue unité du monde hellénistique est évidemment un leurre, que ce soit en matière monétaire ou dans d'autres domaines. Enfin, ni Rostovtseff, ni Finley n'étaient familiers avec la monnaie. Or, des études sur les monnaies de la période fournissent des données qui doivent être prises en compte dans la réflexion, car les corpus monétaires par coins déjà établis constituent de véritables séries documentaires permettant une estimation de la production de plusieurs ateliers (cf. annexe 1). Il n'est pas encore possible de proposer des résultats globaux, mais cela viendra. Et, dès à présent, les analyses élémentaires de monnaies de ces séries et l'étude des trésors et des monnaies de fouille apportent des informations sur l'évolution de la production et sur la circulation monétaires.

Pour ce qui concerne la basse époque hellénistique ( $\mathrm{II}^{\mathrm{e}}-\mathrm{I}^{\mathrm{er}} \mathrm{s}$.), les études récentes sur plusieurs régions donnent à penser que le tableau général présenté par Rostovtseff doit être amendé. J'évoquerai ici le cas de la Grèce méridionale, et plus particulièrement du Péloponnèse.

\section{HISTOIRE MONÉTAIRE DU PÉLOPONNÈSE À L'ÉPOQUE HELLÉNISTIQUE}

Pendant la haute époque hellénistique (fin du $\mathrm{IV}^{\mathrm{e}}-\mathrm{III}^{\mathrm{e}} \mathrm{s}$.), plusieurs cités importantes furent occupées par les Macédoniens (Corinthe) ou gouvernées par des tyrans philomacédoniens. La production monétaire locale paraît alors avoir été très faible. L'essentiel de la masse monétaire était constitué par des monnaies d'argent macédoniennes d'étalon attique de Philippe II, d'Alexandre et de leurs successeurs, monnaies dont le rôle international évoque celui du dollar à notre époque. A cela s'ajoutaient des monnaies d'étalon régional (l'étalon éginétique), en majorité des fractions de $\pm 3 \mathrm{~g}$. La situation a totalement changé à la basse époque hellénistique ( $\mathrm{II}^{\mathrm{e}}-\mathrm{I}^{\mathrm{er}} \mathrm{s}$.). Les monnaies royales d'étalon attique ont alors disparu des trésors au profit de monnaies péloponnésiennes et athéniennes. Dans le cas du monnayage d'Athènes, 1136 coins de droit sont attestés (avec un coin de droit, on pouvait frapper jusqu'à 20 à 30000 monnaies) pour les tétradrachmes stéphanéphores (avec une couronne au revers) de \pm 17 g. d'argent frappés entre 185 (?) et $45(?)^{4}$. Dans le Péloponnèse, la production de monnaies d'argent et de

3 Andreau et Etienne (1984).

4 Thompson (1961). La datation précise de ces monnaies est très discutée, mais elles appartiennent à la basse époque hellénistique. 
bronze d'étalon attique et d'étalon symmachique (éginétique réduit) a augmenté de manière spectaculaire dès le début du $\mathrm{II}^{\mathrm{e}} \mathrm{s}$., et s'est poursuivie au même niveau jusqu'au milieu du $\mathrm{I}^{\mathrm{er}} \mathrm{s}$. av. n. è., à en juger par les corpus déjà constitués :

Sparte : 19 coins de droit au moins ont été utilisés pour les monnaies d'argent du $\mathrm{III}^{\mathrm{e}}$ s. contre 87 pour celles des $\mathrm{II}^{\mathrm{e}}-\mathrm{I}^{\mathrm{er}}$ s. ; pour ce qui concerne les monnaies de bronze, on passe de 24 coins de droit attestés au $\mathrm{III}^{\mathrm{e}} \mathrm{s}$. à 460 pour la production des $\mathrm{II}^{\mathrm{e}}-\mathrm{I}^{\mathrm{er}} \mathrm{s} .^{5}$

Messène : 9 coins de droit sont attestés (10-15 ont pu être utilisés selon les estimations statistiques) pour les monnaies d'argent des $\mathrm{IV}^{\mathrm{e}}-\mathrm{III}^{\mathrm{e}}$ s. contre 107 (110-140) pour celles des $\mathrm{II}^{\mathrm{e}}-\mathrm{I}^{\mathrm{er}} \mathrm{s}$. (triobole du $\mathrm{II}^{\mathrm{e}}$ s. à types civiques, photo $\left.n^{\circ} 1\right)$; pour les monnaies de bronze, on passe de 43 coins de droit (45-65) à $283(300-400)^{6}$.

Cette augmentation de la production est liée à la disparition de la monarchie macédonienne et de son monnayage pendant la première moitié du II ${ }^{\mathrm{e}} \mathrm{s}$. Il n'y a pas eu de vide monétaire, car le relais a été pris par des cités. De plus, pendant cette période, les cités du Péloponnèse se sont groupées dans un Etat fédéral, le koinon achaien. L'historien Polybe, qui était aussi un dirigeant achaien, écrit (II, 37) qu'au sein de la région « l'unification est devenue si complète qu'il ne s'agit plus seulement d'une association de peuples alliés et amis, mais que tous usent des mêmes lois, des mêmes poids, des mêmes mesures et de la même monnaie. »

Ce monnayage fédéral achaien se compose de petites monnaies d'argent de 2,3 g. qui sont des trioboles d'étalon symmachique. Ces monnaies figurent au droit une tête de Zeus lauré (le Zeus fédéral) et au revers les initiales du koinon achaien (AX pour Achaiôn) dans une couronne, avec les symboles propres à chaque cité (par exemple sur les monnaies fédérales de Sparte, les bonnets des Dioscures, cf. la photo $\mathrm{n}^{\circ} 2$ ). Il y a eu en outre une émission de monnaies de bronze. Ce monnayage achaien a été très abondant (probablement au moins 1 à 2 milliers de coins de droit), sans qu'il soit possible de quantifier encore la production en l'absence de corpus par coins. Les analyses élémentaires pratiquées sur une centaine de trioboles péloponnésiens du Cabinet des Médailles de la BNF au Centre Babelon dirigé par Jean-Noël Barrandon donnent toutefois des informations dignes d'intérêt (cf. le schéma p. 22).

- III' s. : 13 monnaies d'Argos et d'Elis

- Agrinion Early et Late : 34 monnaies aux types achaiens d'émissions représentées dans le trésor d'Agrinion de la fin du $\mathrm{II}^{\mathrm{e}} \mathrm{s}$.

- Later : 33 monnaies aux types achaiens d'émissions postérieures à celles représentées dans le trésor d'Agrinion (extrême fin du $\mathrm{II}^{\mathrm{e}} \mathrm{s}$. $-\mathrm{I}^{\mathrm{er}} \mathrm{s}$. av. n. è.).

- Types propres post. à Agrinion : 26 monnaies d'émissions à types civiques, contemporaines des précédentes (extrême fin du $\mathrm{II}^{\mathrm{e}} \mathrm{s}$. $-\mathrm{I}^{\mathrm{er}} \mathrm{s}$. av. n. è.).

Les poids et les poids de métal fin (poids d'argent pur) des trioboles du koinon achaien analysés sont supérieurs à ceux des trioboles frappés au $\mathrm{III}^{\mathrm{e}} \mathrm{s}$. dans la

\footnotetext{
5 Grunauer-von Hoerschelmann (1978).

6 Grandjean (2003).
} 
Trioboles péloponésiens de l'époque hellénistique

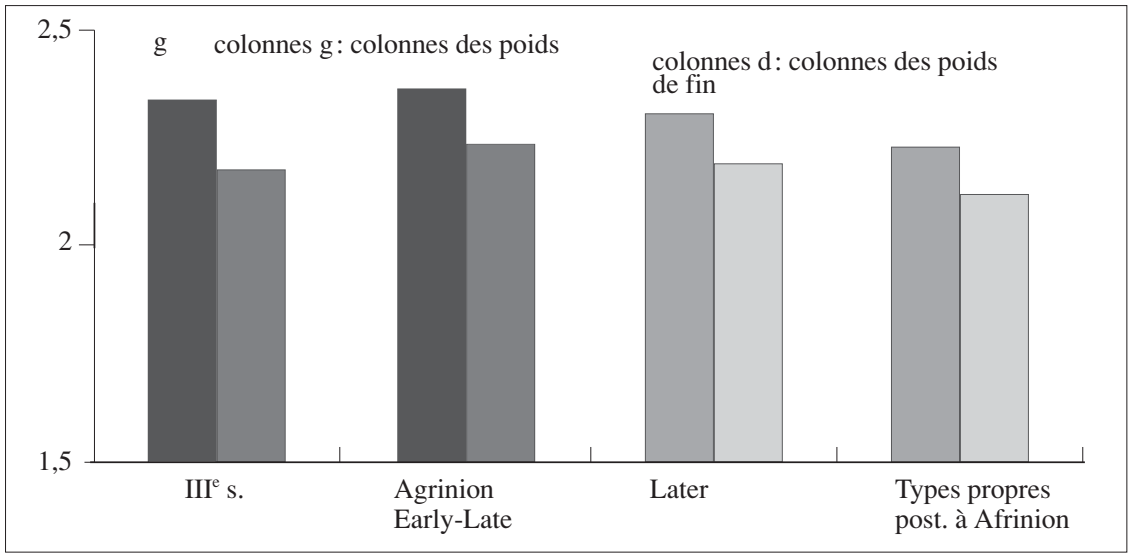

région. Faute de ressources locales en argent, les Achaiens ont dû, comme cela a sans doute été bien souvent le cas, refondre des monnaies royales et d'anciennes monnaies locales pour battre monnaie ${ }^{7}$.

En 146 av. n. è., le koinon achaien fut vaincu et démantelé par Rome et la région passa de fait sous l'autorité du gouverneur de Macédoine. Un koinon achaien, qui ne regroupait que quelques cités, fut reconstitué quelques années plus tard. La province d'Achaïe fut probablement constituée en 27 av. n. è. L'étude de la circulation donne à penser que la production de monnaies d'argent des Etats du Péloponnèse (monnaies à types civiques et monnaies du koinon achaien) ne s'est pas interrompue en 146, mais s'est poursuivie jusqu'à la bataille d'Actium en 31 av. n. è. La pénurie d'argent n'est guère perceptible avant les années 80 et paraît liée aux ponctions des imperatores pendant les guerres civiles. Les monnaies qui doivent être les dernières émissions de monnaies d'argent du Péloponnèse (l'émission d'Aigion à types achaiens au nom d'ARISTODAMOS et l'émission à types civiques de Patras au nom de DAMASIAS ${ }^{8}$ ) sont celles dont le poids de fin est le plus faible : le poids de fin moyen des six monnaies analysées d'Aigion est de 2,05 g., et celui des six monnaies de Patras est de 2,09 g. Le pourcentage de cuivre de ces pièces dépasse souvent $5 \%$. On retrouve des pourcentages de cuivre comparables pour les six deniers analysés des émissions légionnaires frappés par Antoine peu avant Actium, vraisemblablement en Grèce.

7 Il s'agissait sans doute là d'une pratique fréquente, mais dont la quantification est évidemment impossible : pour gagner du temps et économiser les coûts de frappe, les Etats grecs dépourvus de ressources métalliques surfrappaient aussi des monnaies étrangères à leurs propres types (on distingue souvent quelques éléments des types initiaux) ou se contentaient de frapper une contremarque validant d'anciennes monnaies, cf. Le Rider (1975).

8 Warren (1997), Kroll (1997). 
Pourcentages d'argent et de cuivre de 18 monnaies du Cabinet des Médailles de la BNF analysées au Centre Babelon $($ ag $=$ argent $; \mathrm{cu}=$ cuivre $)$

\begin{tabular}{|c|c|c|c|c|c|c|}
\hline \multicolumn{7}{|c|}{ Deniers légionnaires } \\
\hline $\begin{array}{l}\text { élément } \\
\mathrm{Ag} \\
\mathrm{Cu}\end{array}$ & $\begin{array}{l}\text { A4405 } \\
88,4 \\
10,19\end{array}$ & $\begin{array}{l}\text { A4320 } \\
84,1 \\
15,3\end{array}$ & $\begin{array}{l}\text { A4347 } \\
93,6 \\
05,0\end{array}$ & $\begin{array}{l}\text { A4376 } \\
86,5 \\
12,6\end{array}$ & $\begin{array}{l}\mathbf{9 6 4} \\
89,8 \\
08,9\end{array}$ & $\begin{array}{l}\mathbf{2 3 6 9} \\
94,804,1\end{array}$ \\
\hline \multicolumn{7}{|c|}{ Aigion (Aristodamos) } \\
\hline & $\begin{array}{l}\mathbf{3} \\
90,6 \\
08,3\end{array}$ & $\begin{array}{l}\text { NC1 } \\
86,6 \\
12,5\end{array}$ & $\begin{array}{l}\text { D.1971 } \\
91,5 \\
07,5\end{array}$ & $\begin{array}{l}\text { D.1972 } \\
89,9 \\
09,3\end{array}$ & $\begin{array}{l}\mathbf{2 7 8 0 2} \\
93,1 \\
06,0\end{array}$ & $\begin{array}{l}1 \\
91,8 \\
07,6\end{array}$ \\
\hline \multicolumn{7}{|c|}{ Patras (Damasias) } \\
\hline $\begin{array}{l}\text { élément } \\
\mathrm{Ag} \\
\mathbf{C u}\end{array}$ & $\begin{array}{l}\mathbf{1 1 9 2} \\
87,9 \\
10,7\end{array}$ & $\begin{array}{l}\text { 1192A } \\
94,6 \\
04,6\end{array}$ & $\begin{array}{l}\text { 1192B } \\
95,4 \\
03,8\end{array}$ & $\begin{array}{l}\text { D.1967 } \\
92,1 \\
06,7\end{array}$ & $\begin{array}{l}\mathbf{1 1 9 0} \\
95,9 \\
03,5\end{array}$ & $\begin{array}{l}\mathbf{1 1 9 1} \\
93,7 \\
05,2\end{array}$ \\
\hline
\end{tabular}

La plus extrême prudence est de rigueur avec les analyses élémentaires; elle s'impose a fortiori dans le cas présent, où le nombre d'exemplaires analysés est réduit. Reste que les analyses des deniers plus anciens et d'autres séries grecques d'époque hellénistique menées avec la même technique soulignent la parenté de composition des monnaies d'Achaïe et des deniers légionnaires. Il est probable que les ultimes monnaies d'argent du Péloponnèse ont été frappées pour financer l'effort de guerre d'Antoine.

Après Actium, la frappe de l'argent s'est interrompue dans la région. Faute de texte stipulant une interdiction par les autorités romaines, et parce que la frappe de l'argent s'est poursuivie dans d'autres régions du monde grec, on peut se demander si cet arrêt n'est pas lié à la pénurie d'argent, incontestable à cette époque, mais aussi à la présence de groupes de Romains utilisant la monnaie romaine et peut-être aussi aux problèmes de change entre monnaies de poids éginétique réduit et deniers. La frappe du bronze s'est poursuivie en revanche jusqu'à l'époque des Sévères.

\section{LES ROMAINS ET LA MONNAIE GRECQUE}

Rome n'a manifestement pas cherché à imposer le denier qui a pénétré très lentement en Grèce à partir du milieu du $\mathrm{I}^{\mathrm{er}} \mathrm{s}$. av. n. è. avec les armées des Guerres civiles et la flotte d'Antoine. Dans les inscriptions, le denier est la monnaie de compte à partir des années 70 av. n. è. à Messène, mais, dans les affranchissements de Delphes, les premières mentions du denier datent du $\mathrm{I}^{\mathrm{er}} \mathrm{s}$. et des comptes en monnaies grecques y sont encore attestés au II ${ }^{\mathrm{e}}$ s., à l'époque d'Hadrien ${ }^{9}$.

De même, le système monétaire romain augustéen en cuivre et en orichalque est loin d'avoir été adopté d'emblée : tandis qu'en Occident, les dénominations

\footnotetext{
9 Migeotte (1997), Mulliez (1997).
} 
romaines ont été majoritairement utilisées après adaptation aux usages locaux, l'Orient a connu une grande variété de modules et de poids. Des monnaies frappées au $\mathrm{II}^{\mathrm{e}} \mathrm{s}$. ont continué à circuler dans le Péloponnèse après avoir été validées par des contremarques (monnaie de Messène portant une contremarque au type d'Octave-Auguste, photo $n^{\circ} 3$ ). En outre, les monnaies frappées après la conquête par les cités, qui font rarement référence à l'autorité romaine par leurs types et leurs légendes, avaient les mêmes modules et étaient dans les mêmes alliages qu'auparavant (ce sont des monnaies en bronze).

Les colonies romaines de la région (Dymè, Corinthe, Patras) n'ont pas cherché à imposer le système monétaire romain et se sont souvent adaptées aux traditions locales : ainsi, Corinthe frappait dans la seconde moitié du $\mathrm{I}^{\mathrm{er}} \mathrm{s}$. avant $\mathrm{n}$. è. 4 dénominations de bronze de même module que les pièces de la cité d'Aigion en 32. Les choses ne changèrent guère après la réforme du système monétaire par Auguste : Patras et Corinthe frappèrent l'as augustéen, mais aussi d'autres dénominations non conformes au système augustéen qui permettaient un change facile avec les monnaies locales.

\section{Système monétaire de bronze augustéen ${ }^{10}$}

- Monnaies en orichalque (laiton) :

- sesterce de $25 \mathrm{~g} .=$ quatre $a s$;

- dupondius de 12,5 g. = deux as ;

- Monnaies en cuivre :

- as de $11,27 \mathrm{~g}$. ;

- semis de 4,8 g. = un demi as ;

- quadrans de 3,10 g. = un quart d'as.

\section{Monnaies péloponnésiennes de l'époque des Julio-Claudiens}

Messène :

- bronzes aux types d'Héraclès et de la massue : $\pm 3 \mathrm{~g}$. et $\pm 16 \mathrm{~mm}$ (diamètre du flan);

- bronzes aux types de Messène et d'autres divinités : $\pm 6 \mathrm{~g}$. et \pm $18 \mathrm{~mm}^{11}$.

Patras (colonie romaine fondée par Auguste en 14 av. n. è.) :

- Règne d'Auguste : monnaies de bronze de $\pm 6,46$ g. et $\pm 20 \mathrm{~mm}$;

- Règne de Tibère : as en cuivre de $\pm 9,78 \mathrm{~g}$. et $\pm 25 \mathrm{~mm}$;

- Règne de Claude : as en cuivre de $\pm 9,52 \mathrm{~g}$. et $\pm 25 \mathrm{~mm}$; monnaie en cuivre de $\pm 12,14 \mathrm{~g}$. et $\pm 27 \mathrm{~mm}^{12}$.

\footnotetext{
10 Amandry (1997).

${ }^{11}$ Grandjean (sous presse).

12 Amandry (1997).
} 
Sparte :

- Dynaste Euryclès (31-2 av. n. è.) : monnaies de bronze de $\pm 2,5$ g. et $\pm 15 \mathrm{~mm}, \pm 3,05 \mathrm{~g}$. et $\pm 16 \mathrm{~mm}, \pm 5,64 \mathrm{~g}$. et $\pm 20 \mathrm{~mm}, \pm 10,69 \mathrm{~g}$. et $\pm 27 \mathrm{~mm}$;

- Dynaste Lako (2 av. n. è. - 31) : monnaies de bronze de $\pm 4,2$ g. et $\pm 15 \mathrm{~mm}, \pm 5,74 \mathrm{~g}$. et $\pm 20 \mathrm{~mm}, \pm 7,5 \mathrm{~g}$. et $\pm 20 \mathrm{~mm}^{13}$.

Sicyone :

- Règne de Néron : monnaies de bronze de $\pm 7,65 \mathrm{~g}$. et $\pm 20 \mathrm{~mm}, \pm$ $6,8 \mathrm{~g}$ et $\pm 20 \mathrm{~mm}, \pm 7 \mathrm{~g}$ et $\pm 20 \mathrm{~mm}$, etc. ${ }^{14}$
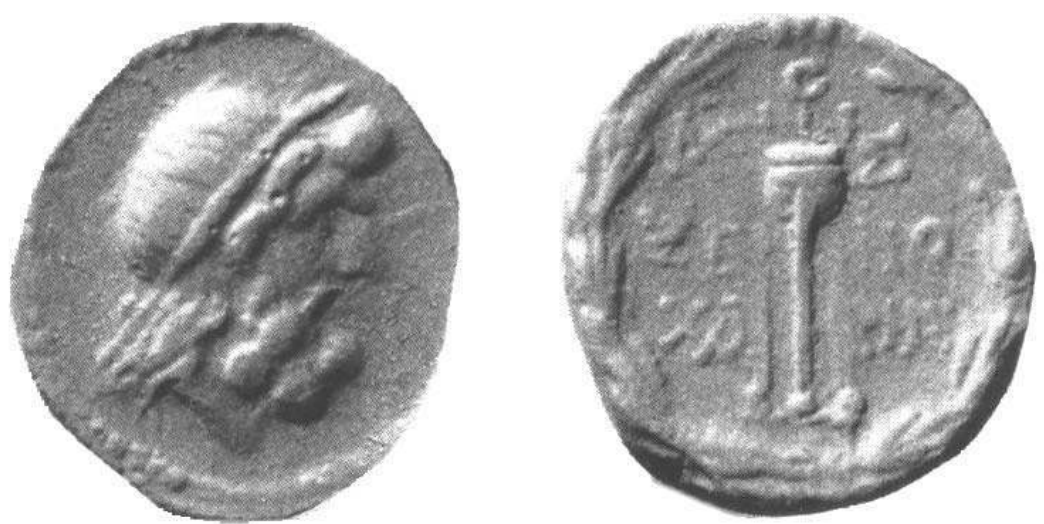

1. Messène : Paris K 2819 -triobole en argent à types civiques (Ir s. av. n.è.) -au ??? Zeus, au revers trépied et lettres et monogrammes.
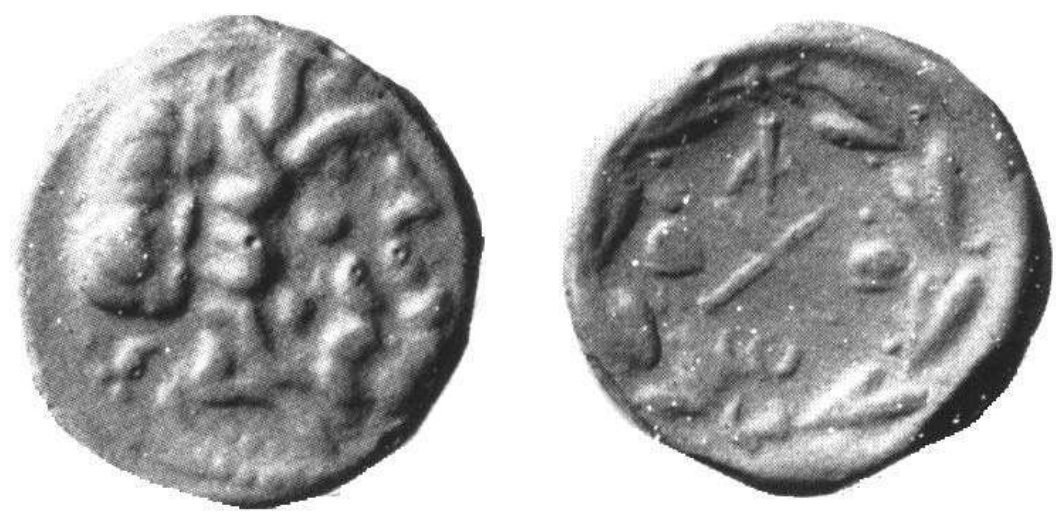

2. Sparte : Paris Delepierre 2001 - triobole en argent aux types achaiens - au revers, bonnets des Dioscures (II ou Ir s. av. n. ̀̀.) et monogramme.

${ }^{13}$ Grunauer (1978).

${ }^{14}$ Amandry, Burnett, Pau Ripollès (1992) où l'on trouvera de nombreux autres exemples. 


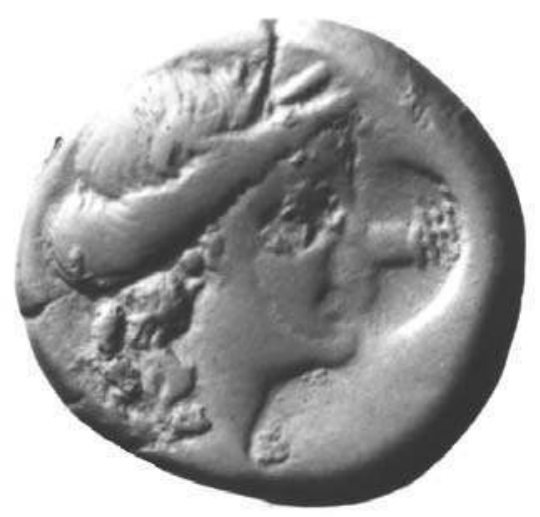

3. Messène : Paris 1748 - monnaie de bronze du II ${ }^{e}$ s. (la contremarque au type d'Octave-Auguste a été apposée horizontalement en haut du droit) au type de Déméter.

Il a fallu en réalité attendre le III ${ }^{\mathrm{e}} \mathrm{s}$. pour que s'exprime une véritable volonté d'uniformisation avec l'émission de bronze péloponnésienne frappée sous les Sévères par une quarantaine de cités, vraisemblablement pour financer une expédition de Caracalla en Syrie. Ces monnaies figurent un buste impérial et sont dans un alliage qui n'est pas conforme aux traditions locales, avec un taux de plomb très élevé et un taux de cuivre bien plus faible qu'auparavant.

Si l'on met à part la période des Guerres civiles du $\mathrm{I}^{\mathrm{er}} \mathrm{s}$. av. n. è., où des monnayages civiques grecs ont été en réalité frappés pour financer l'effort de guerre des imperatores (c'est le cas notamment de plusieurs émissions de stéphanéphores attiques et probablement aussi de trioboles péloponnésiens), les interventions des Romains en matière monétaire dans la région paraissent s'être limitées à imposer des taux de change entre monnaies grecques et romaines légèrement favorables à la monnaie romaine, avec notamment l'équivalence entre la drachme attique ( \pm 4,2 g.) et le denier (3,9 g.), puis le diorthôma (redressement) imposé par Auguste, qui devait fixer l'équivalence entre le statère de Thessalie de $\pm 6 \mathrm{~g}$. et 1,5 denier $^{15}$.

A priori, ce manque d'unité invite à en rabattre sur la rigueur logique et la volonté d'uniformisation que l'on attribue souvent aux Romains et à se demander si à leurs yeux existait un véritable lien entre souveraineté et monnayage. Reste que la latitude laissée en la matière aux Grecs (et autres peuples) était limitée : ils n'avaient pas le droit de frapper l'or, apanage impérial. De plus, les monnaies civiques en bronze étaient destinées surtout à une utilisation locale et aux paiements fiscaux. Elles ne sortaient guère du Péloponnèse que pour être refondues à Rome. En outre, le système présentait des avantages économiques et politiques non négligeables pour les Romains. Ils économisaient ainsi les frais de frappe de ces émissions locales (préparation des coins, perte de métal lors de la refonte, entretien des graveurs et des ouvriers de l'atelier, etc.). De plus et surtout,

\footnotetext{
15 Helly (1997).
} 
le système respectait les traditions d'autonomia (le droit de battre monnaie à ses types et à son nom était un élément de l'autonomia, comme le libre fonctionnement de toutes les institutions civiques) auxquelles les cités grecques étaient passionnément attachées, manifestant bruyamment leur mécontentement dès que ces traditions étaient menacées par une cité voisine ou par un magistrat romain.

\section{LE DÉVELOPPEMENT \\ DU MONNAYAGE DE BRONZE \\ ET LA PRÉTENDUE CRISE DE LA BASSE ÉPOQUE HELLÉNISTIQUE}

Dès la Guerre du Péloponnèse (431-405), longue et coûteuse, la monnaie de bronze, déjà connue en Sicile et à Olbia, a été frappée par plusieurs cités grecques lorsque l'argent manquait. Dans les cités d'ancienne tradition monétaire (Athènes, Argos et Sicyone notamment), cette monnaie au caractère largement fiduciaire ne s'est pas imposée d'emblée. Le théâtre d'Aristophane atteste des réticences des Athéniens : «Et le décret sur les monnaies de bronze, ne te le rappellestu pas ? - Ce fut même un désastre pour moi, que cette monnaie ! Car, un jour que je revenais de vendre des raisins, je m'en retournai la bouche pleine de pièces de bronze, puis je m'en fus à l'agora pour acheter de la farine ; comme je présentai mon sac, le héraut cria : Défense à quiconque d'accepter à l'avenir aucune pièce de bronze; l'argent seul aura cours » (Assemblée des femmes 815-822). «Ainsi en va-t-il pour les citoyens : ceux que nous savons de bonne naissance, raisonnables, justes, probes et honnêtes, formés aux exercices de la palestre, aux chœurs et à la musique, ceux-là nous les outrageons et nous faisons servir à tous nos usages les pièces de bronze, je veux dire des étrangers, des roux, des vauriens fils de vauriens, nouveaux venus dont la cité n'eût pas voulu facilement, sans contrôle, même pas comme victimes expiatoires » (Grenouilles 727-733) (traduction M.-J. Alfonsi). Ailleurs dans le Péloponnèse, dans les cités libérées de la domination spartiate (et de l'interdiction de battre monnaie qui en résultait) par la bataille de Leuctres (371), des monnaies d'argent et de bronze ont été frappées de concert dès les premières émissions, le bronze étant d'emblée le métal des fractions d'usage local d'une valeur inférieure à celle de l'obole (Messène, Hermione, etc. $)^{16}$. Malgré les résistances locales, le bronze monnayé s'est très largement répandu dans les cités et royaumes du monde grec dès la seconde moitié du $\mathrm{IV}^{\mathrm{e}} \mathrm{s}$.

Les premières monnaies de bronze étaient lourdes et comportaient une part importante d'étain, métal importé et cher. Très vite toutefois - les analyses élémentaires l'attestent -, les ateliers ont diminué la part de l'étain au profit de celle du plomb, bien moins coûteux et très répandu en Grèce (ainsi, l'argent du Laurion attique provenait de la galène argentifère) : la part de fiduciarité de ces monnaies (et le profit des Etats émetteurs) a manifestement augmenté à mesure que diminuaient les réticences des usagers. A l'origine, la plupart des cités frappèrent une dénomination de bronze, mais elles se dotèrent le plus souvent dès le $\mathrm{IV}^{\mathrm{e}} \mathrm{s}$. de

\footnotetext{
${ }^{16}$ Grandjean (1998).
} 
systèmes de 2 ou 3 dénominations, voire davantage, pour toutes les fractions, cessant toutes peu à peu de frapper l'obole en métal précieux.

Le développement du bronze monnayé dans le monde grec est donc bien plus ancien que ne le pensait Rostovtseff. Ce qui paraît caractéristique de l'époque hellénistique et sans doute lié à des crises d'approvisionnement en argent est la frappe de dénominations en bronze de valeur plus élevée qu'auparavant : des trioboles athéniens en bronze furent probablement frappés à la fin du $\mathrm{III}^{\mathrm{e}} \mathrm{s}$. et des inscriptions béotiennes du $\mathrm{II}^{\mathrm{e}} \mathrm{s}$. font état de drachmes de bronze ${ }^{17}$.

La montée en puissance du bronze est allée de pair aussi avec la monétarisation progressive des échanges et singulièrement du commerce de détail comme l'attestent les inscriptions de l'époque hellénistique donnant les tarifs à la mine (environ 400 grammes) imposés par l'Etat pour différents poissons et abats : les prix sont exprimés en oboles et en chalques (monnaies de bronze) dans des inscriptions d'Athènes et d'Akraiphia (Béotie), et seulement en chalques dans une inscription de Delphes ${ }^{18}$.

Pour ce qui concerne le Péloponnèse de la basse époque hellénistique, les campagnes récentes de prospection archéologique ont mis en lumière que des changements étaient intervenus dans l'exploitation de la région sous l'autorité du koinon achaien, changements qui se sont poursuivis pendant le Haut-Empire avec notamment la tendance au développement de grandes exploitations au détriment des petites en faire-valoir direct. La période a été marquée par une intensification et une spécialisation agricoles (ainsi, le développement de la culture du lin dans l'ouest du Péloponnèse, traité après la récolte à Patras), tandis que se développait l'élevage extensif sur les terres laissées en friches. Parmi les grands propriétaires, on trouvait évidemment des Rhomaioi (Italiens et Romains), mais surtout des membres des élites locales ${ }^{19}$.

L'archéologie comme la numismatique invitent donc à adopter pour l'histoire économique de cette région un autre modèle et d'autres césures chronologiques que ceux proposés par Rostovtseff. La basse époque hellénistique y correspond à une période de prospérité où la politique de développement économique menée avec un monnayage abondant au profit des élites locales a anticipé la politique romaine.

Dans d'autres régions de Grèce continentale et de Grèce d'Asie, des cités et des Etats fédéraux ont pris le relais des royaumes hellénistiques en matière monétaire, avec l'aval des Romains. Rostovtseff paraît surtout avoir fondé sa vision de la situation monétaire de la période sur le témoignage des sources textuelles relatives à l'Egypte. Or, les travaux récents sur les monnaies des Ptolémées donnent à penser que la pénurie monétaire lagide elle-même est assez largement une légende.

\section{BIBLIOGRAPHIE}

Alcock, Susan E., Graecia Capta The landscapes of Roman Greece, Cambridge, 1993.

Amandry, Michel, Burnett, Andrew, Ripolles, Pau, Roman Provincial Coinage, I, Londres-Paris, 1992.

\footnotetext{
${ }^{17}$ Grandjean (1995), Kroll (1993).

18. Vatin (1966), Salviat et Vatin (1971), Steinhauer (1994).

19 Alcock (1993).
} 
Amandry, Michel, « Les colonies romaines ont-elles été le vecteur de l'introduction des dénominations romaines au pourtour de la Méditerranée? », Colloque « De la drachme au denier », Topoi 7/1, 1997, pp. 137-148.

Andreau, Jean et Etienne, Roland, « Vingt ans de recherches sur l'archaïsme et la modernité des sociétés antiques », Revue des Etudes Anciennes, 86, 1984, pp. 55-83.

Finley, Moses I., Les Anciens Grecs, Paris, 1971.

Grandjean, Catherine, «Les comptes de Pompidas (IG VII 2426). Drachmes d'argent symmachique et drachmes de bronze », BCH 119, 1995, pp. 1-26.

Grandjean, Catherine, «La valeur des monnaies de bronze du Péloponnèse », Revue Numismatique, 153, 1998, pp. 31-40.

Grandjean, Catherine, Les Messéniens de 370/369 au I er siècle de notre ère. Monnayages et histoire, BCH Supplément 44, 2003.

Grunauer-von Hoerschelmann, Susanne, Die Münzprägung der Lakedaimonier, Berlin, 1978.

Helly, Bruno, « Le diorthôma d'Auguste fixant la conversion des statères thessaliens en deniers, une situation de passage à la monnaie unique », Colloque « De la drachme au denier », Topoi 7/1, 1997, pp. 63-91.

Kroll, John H, The Athenian Agora The Greek Coins, Princeton, 1993.

Kroll, John H, «Traditionalism vs Romanization », Colloque « De la drachme au denier », Topoi 7/1, 1997, pp. 123-136.

Le Rider, Georges, «Contremarques et surfrappes dans l'Antiquité grecque », Numismatique antique Problèmes et méthodes, Annales de l'Est, 44, 1975.

Migeotte, Léopold, « La date de l'oktôbolos eisphora de Messène », Colloque « De la drachme au denier », Topoi, 7/1, 1997, pp. 51-61.

Mulliez, Dominique, « Le denier dans les actes d'affranchissement delphiques », Colloque « De la drachme au denier », Topoi, 7/1, 1997, pp. 93-102.

Rostovtseff, Michel Ivanovic, Histoire économique et sociale du monde hellénistique, Paris, Robert Laffont, 1989.

Salviat, François et Vatin, Claude, Inscriptions de Grèce centrale, Paris, 1971, pp. 95-110.

Steinhauer, Georges, «L'inscription agoranomique du Pirée », BCH 118 (1994), pp. 53-68.

Thompson, Margaret, The New Style Silver Coinage of Athens, New York, 1961.

Vatin, Claude, « Un tarif des poissons à Delphes », BCH 90, 1966, pp. 274-280.

Warren, Jennifer, « After the Boehringer Revolution », Colloque « De la drachme au denier », Topoi, 7/1, 1997, pp. 109-114.

\section{Annexe 1 \\ LA CONSTITUTION DES CORPUS MONÉTAIRES}

L'écrasante majorité des monnaies grecques a été frappée. Le flan (pastille de métal) était posé sur le coin de droit (morceau de métal très dur où était gravé le type monétaire à frapper au droit de la pièce) préalablement fixé dans une enclume ; on positionnait dessus le coin de revers sur lequel était donné le coup de marteau qui imprimait sur le flan les types gravés sur les coins.

L'étude des coins monétaires est le fondement de la constitution des corpus et de la recherche numismatique depuis les travaux pionniers du numismate suisse F. Imhoof-Blumer. Il s'agit, à partir de l'examen du plus grand nombre possible de 
monnaies d'un atelier, de déterminer les identités de coins, puis le nombre de coins de droit et de revers utilisés (les types gravés n'étaient jamais identiques).

Les coins de droit et de revers ne s'usaient pas à la même vitesse. Le coin de droit était plus éloigné de l'impact du coup de marteau que le coin de revers ; en outre, le plan de frappe était toujours identique pour le coin de droit, mais pas pour le coin de revers tenu à la main. En général, les ateliers utilisaient donc davantage de coins de revers que de coins de droit et ces derniers étaient le plus souvent associés à plusieurs coins de revers. A partir de là, il est possible de reconstituer la chronologie relative de chaque émission (épisode de frappe caractérisé par des types et/ou surtout par des marques de contrôle inscrites au revers, noms ou symboles de monétaires) voire même, lorsque des coins de droit ont été utilisés pour plusieurs émissions, la chronologie relative de plusieurs séries. A en juger par les inscriptions de Delphes et par des expériences modernes, il était possible de frapper jusqu'à environ 30000 monnaies par coin de droit. 